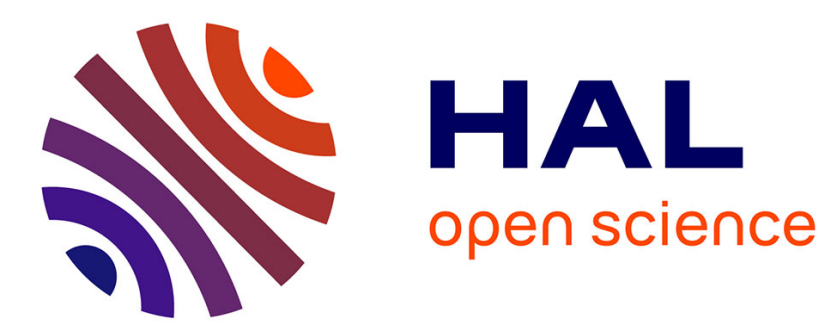

\title{
Lift forces in granular media
}

François Guillard, Yoël Forterre, Olivier Pouliquen

\section{To cite this version:}

François Guillard, Yoël Forterre, Olivier Pouliquen. Lift forces in granular media. Physics of Fluids, 2014, 26 (4), pp.043301. 10.1063/1.4869859 . hal-00997840

\section{HAL Id: hal-00997840 https://hal.science/hal-00997840}

Submitted on 29 May 2014

HAL is a multi-disciplinary open access archive for the deposit and dissemination of scientific research documents, whether they are published or not. The documents may come from teaching and research institutions in France or abroad, or from public or private research centers.
L'archive ouverte pluridisciplinaire HAL, est destinée au dépôt et à la diffusion de documents scientifiques de niveau recherche, publiés ou non, émanant des établissements d'enseignement et de recherche français ou étrangers, des laboratoires publics ou privés. 


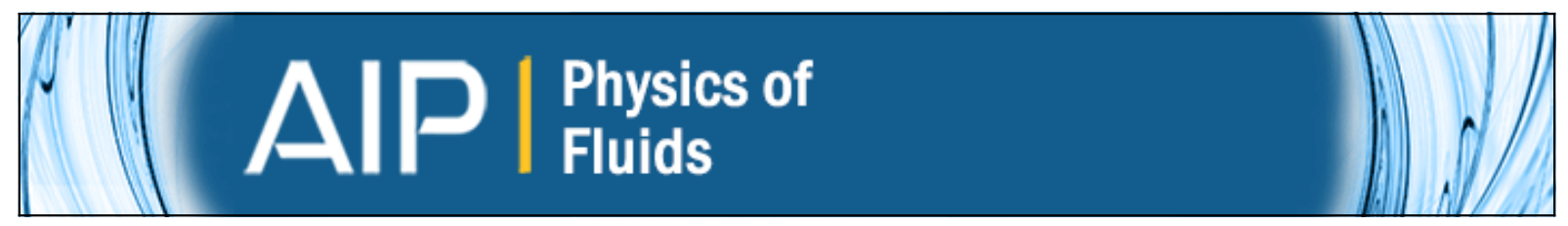

\section{Lift forces in granular media}

François Guillard, Yoël Forterre, and Olivier Pouliquen

Citation: Physics of Fluids (1994-present) 26, 043301 (2014); doi: 10.1063/1.4869859

View online: http://dx.doi.org/10.1063/1.4869859

View Table of Contents: http://scitation.aip.org/content/aip/journal/pof2/26/4?ver=pdfcov

Published by the AIP Publishing

\section{Articles you may be interested in}

Granular force on objects and correlation length: Drag coefficient enhancement in low Froude number flow regimes

AIP Conf. Proc. 1542, 617 (2013); 10.1063/1.4812007

Scaling laws in granular flow and pedestrian flow

AIP Conf. Proc. 1542, 157 (2013); 10.1063/1.4811891

The unsteady drag force on a cylinder immersed in a dilute granular flow

Phys. Fluids 18, 043301 (2006); 10.1063/1.2191907

Numerical simulation of two-dimensional steady granular flows in rotating drum: On surface flow rheology

Phys. Fluids 17, 103303 (2005); 10.1063/1.2063347

Radial mixing of granular materials in a rotating cylinder: Experimental determination of particle self-diffusivity Phys. Fluids 17, 013101 (2005); 10.1063/1.1825331

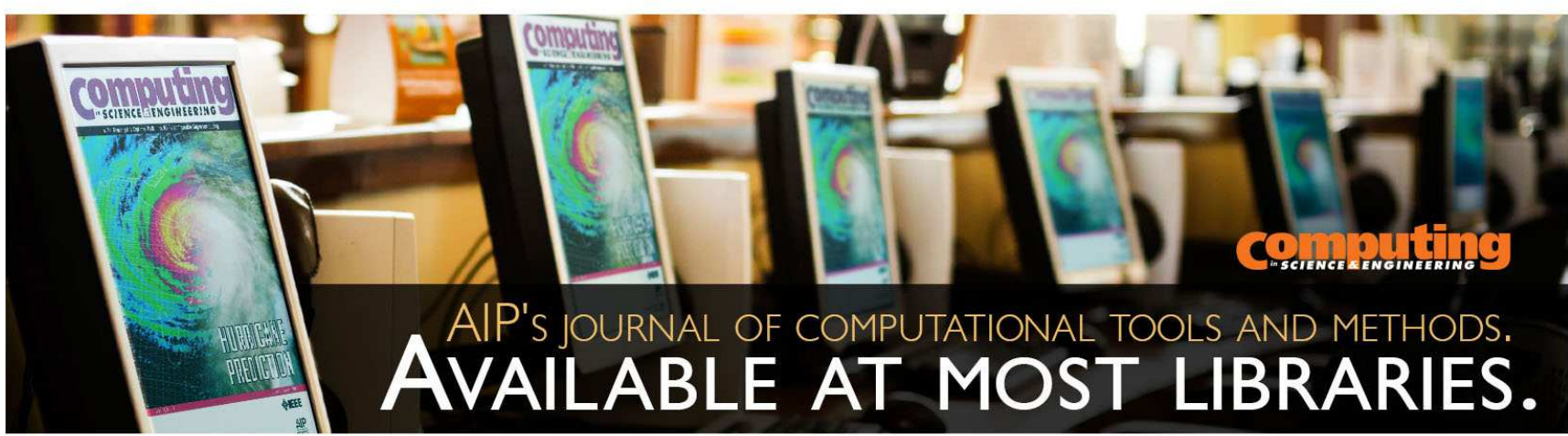




\title{
Lift forces in granular media
}

\author{
François Guillard, ${ }^{\text {a) }}$ Yoël Forterre, ${ }^{\text {b) }}$ and Olivier Pouliquen ${ }^{c)}$ \\ Aix-Marseille Université, CNRS, IUSTI UMR 7343, 5 rue Enrico Fermi, \\ 13453 Marseille Cedex 13, France
}

(Received 20 September 2013; accepted 17 March 2014; published online 3 April 2014)

\begin{abstract}
The paper presents an experimental and numerical study of the forces experienced by a cylinder moving horizontally in a granular medium under gravity. Despite the symmetry of the object, a strong lift force is measured. Whereas the drag force increases linearly with depth, the lift force is shown to saturate at depths much greater than the cylinder diameter, and to scale like the buoyancy with a large amplification factor of order 20. The origin of this high lift force is discussed based on the stress distribution measured in discrete numerical simulations. The lift force comes from the gravitational pressure gradient, which breaks the up/down symmetry and strongly modifies the flow around the obstacle compared to the case without pressure gradient. (C) 2014 AIP Publishing LLC. [http://dx.doi.org/10.1063/1.4869859]
\end{abstract}

\section{INTRODUCTION}

Forces experienced by objects moving in a fluid is a classical problem in fluid mechanics. It has been extensively studied in the case of a newtonian fluid. The case of non-newtonian fluids has attracted attention more recently, and researches has been conducted by dragging objects in polymers, ${ }^{1,2}$ colloïdal suspensions, ${ }^{3}$ gels $^{4}$ or foams. ${ }^{5}$ The aim of the work presented in this paper is to investigate the case of an object moving in a granular medium. This problem, which is of practical importance in many applications (stirring, ${ }^{6}$ mining, mixing, problems of impacts, ${ }^{7}$ locomotion in sand $^{8}$ ), is also of fundamental interest to probe the rheology of particulate materials..$^{9,10}$

When studying the forces experienced by an object in a flow, the total force is usually split in two contributions: a drag force parallel to the direction of the mean flow, and a lift force perpendicular to the flow. The drag force in granular media has been studied in detail by many authors in different configurations, with the obstacle dragged either horizontally ${ }^{11,12}$ or vertically as in impact problems. ${ }^{13-15}$ A main result is that at low speeds a quasi static regime exists, for which the drag is independent of the velocity and proportional to the hydrostatic pressure times the surface area of the object. ${ }^{16-19}$ The robustness of this frictional scaling has been tested by changing the geometry of the objects, ${ }^{20}$ by probing the effect of the boundaries of the vessel containing the medium, ${ }^{21}$ by changing the effective gravity ${ }^{22}$ or by varying the initial volume fraction. ${ }^{19}$ At higher velocities such as encountered in impact problems, an inertial contribution is observed in addition to the frictional one, which scales as the square of the velocity. ${ }^{15}$ By contrast, very few studies address the question of the lift force in granular media. Percier et al. ${ }^{23}$ studied the lift experienced by an inclined plate moving at the free surface of a granular medium. The case of an object entirely plunged into the packing has been studied first by Ding et al. ${ }^{24}$ in the quasi-static regime and more recently by Potiguar and Ding $^{25}$ at higher velocities. In their experiments and simulations, Ding et al. ${ }^{24}$ have dragged different objects horizontally under the free surface. They have shown that even for a symmetric object like a cylinder, a strong lift force exists. A main result of their study is that the lift increases linearly with the depth, like the drag force. The authors have proposed a phenomenological model, in order to compute the lift on obstacles of various geometries. They assume that the lift created by each

\footnotetext{
a) francois.guillard@univ-amu.fr

b)yoel.forterre@univ-amu.fr

c) olivier.pouliquen@univ-amu.fr
} 
elementary surface at the surface of the object is the same as a plate moving alone at the same depth and at the same angle of attack. This model successfully helps in understanding the forces developed by sand lizard during its motion ${ }^{26}$ and for designing the foots of robots walking on sand. ${ }^{27}$

The results obtained by Ding et al. ${ }^{24}$ contrast with our common knowledge of lift in fluid mechanics. The observation of a lift on a cylinder shows that in a granular medium no geometrical asymmetry is necessary to measure a lift, which is not true in newtonian or non-newtonian fluids such as polymers, gels or foams. If not from the shape of the object, the top/bottom asymmetry necessary to observe a lift has to come from the material itself. In a granular medium, the pressure gradient induced by gravity could be the source of this asymmetry. The flow of granular media being controlled by a frictional rheology, i.e., the stresses are proportional to the pressure, the response of the medium in presence of gravity is no longer invariant along the vertical. In this case, the lift force should depend on the pressure gradient and not on the depth, like the buoyancy force in fluid mechanics. This seems to contradict the observations of Ding et al. ${ }^{24}$ who report a lift force that increases linearly with depth. However, their experiments being carried out at moderate depth, the presence of the free surface could break the top/bottom symmetry as well. What happens when the object is dragged at larger depths, far from the free surface? Does the lift still increase with depth or saturate as expected from the above symmetry arguments?

Clarifying these issues and investigating in detail how a lift force develops when an object moves in a granular medium is the goal of this study. The paper is organised as follows. Section I is dedicated to the experimental study performed on fixed cylinders buried in a rotating tank filled with glass beads. The setup is described and the measurements for both the drag and lift forces are analysed in a wide range of cylinder size and depth. The observation of a strong lift force independent of the depth is discussed and a scaling law is evidenced. Section II is dedicated to simulations using a discrete element method. The results are shown to be quantitatively in agreement with the experiments and details about the flow perturbation around the obstacle are discussed. Section III is a discussion about the origin of the lift and an attempt to understand the scaling observed in both the experiments and the simulations based on the analysis of the stress distribution around the object. Conclusions and perspectives are given in Sec. IV.

\section{EXPERIMENTS}

\section{A. Experimental setup}

The experimental setup is sketched in Fig. 1. It is composed of a tank $30 \mathrm{~cm}$ in diameter filled with $23 \mathrm{~cm}$ of glass beads $530 \pm 30 \mu \mathrm{m}$ in diameter and density $\rho_{g}=2.5 \mathrm{~g} \mathrm{~cm}^{-3}$. In such a wide container, no Janssen effect is expected to occur. The tank is fixed on a rotating table and rotates around its vertical axis. All our experiments are performed at a rotation speed equal to $0.8 \mathrm{rpm}$, which corresponds to the quasi-static regime for which we have checked that the measured forces are independent of velocity. ${ }^{17}$ The obstacle is a steel cylinder of length $L$ and diameter $D$, which is buried at the centre of the tank, at depth $h$. The cylinder is kept static and horizontal by a $3 \mathrm{~mm}$ vertical rigid rod fixed at the top of the experiment to a torque meter (Meiri CS1). The measured torque $\mathcal{M}$ experienced by the cylinder when the tank is rotating provides a measurement of the drag force using the following relation ${ }^{28}$ (see caption of Fig. 1):

$$
F_{\text {drag }}=\frac{4}{L} \mathcal{M}
$$

The torque meter is itself fixed on a high precision weighting scale (Mettler Toledo XS $6002 \mathrm{~S}$ ), which gives the vertical force experienced by the cylinder, i.e., the lift force $F_{\text {lift }}$ The whole set-up (cylinder, support, and sensors) is fixed on a motorised vertical translation stage to adjust the depth $h$. Note that in order to get information on the forces exerted on the horizontal cylinder only, the contribution of the vertical support alone is systematically subtracted from the total torque and vertical force when computing the drag and lift forces (the contribution of the support represents less than $5 \%$ of the total forces). 


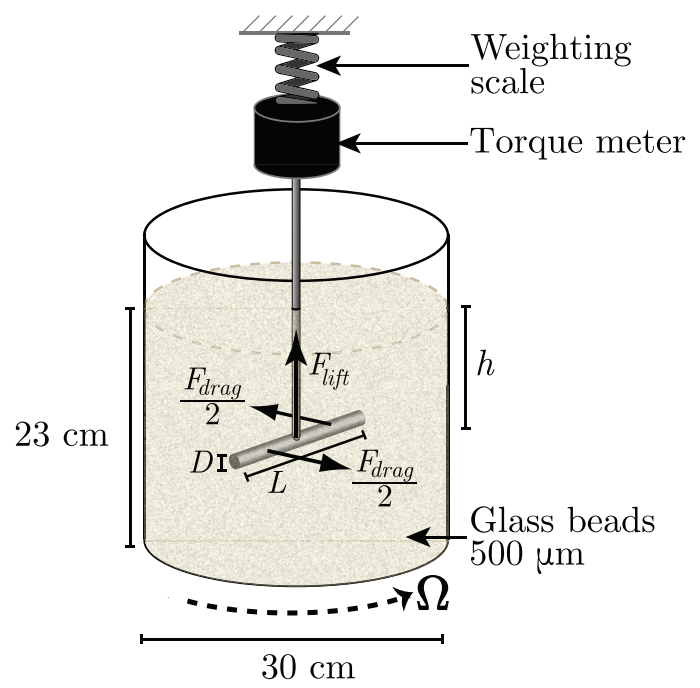

FIG. 1. Sketch of the experiment. A static cylinder is buried in a rotating tank filled with glass beads. A torque meter and a precision weighting scale record the drag and lift forces on the cylinder. Note that each half of the cylinder experiences a force equal to $F_{\text {drag }} / 2$, which implies Eq. (1).

The experimental procedure is the following. The grains are first poured in the tank and stirred by hand. The cylinder is then introduced in the packing and the tank is put in rotation. Figure 2 presents the time evolution of both the drag and lift forces on the cylinder during several rotations. Initially, both the torque and the lift increase and reach a plateau. The drag then dramatically drops after half a rotation, when the cylinder goes through its wake. ${ }^{28}$ This drop of the drag force after half a turn has been studied in detail by Guillard et $a .^{28}$ and comes from the development of a structure in the medium as the cylinder continuously passes through its own wake. Here we focus on the first half-turn, when the medium is not affected by the motion of the cylinder. The forces are then recorded during the first half-rotation only, and time-averaged in the plateau region (Fig. 2). Moreover, to avoid any bias in the structure of the medium, the direction of the rotation is systematically reversed between two successive experiments. A remark is that the measurements obtained just after the tank has been filled fluctuates from one run to another. However, after several runs performed at different depths $h$, the medium no longer evolves, and measurements are then highly reproducible and independent of the filling procedure. All the data presented in the paper are obtained in this steady regime.

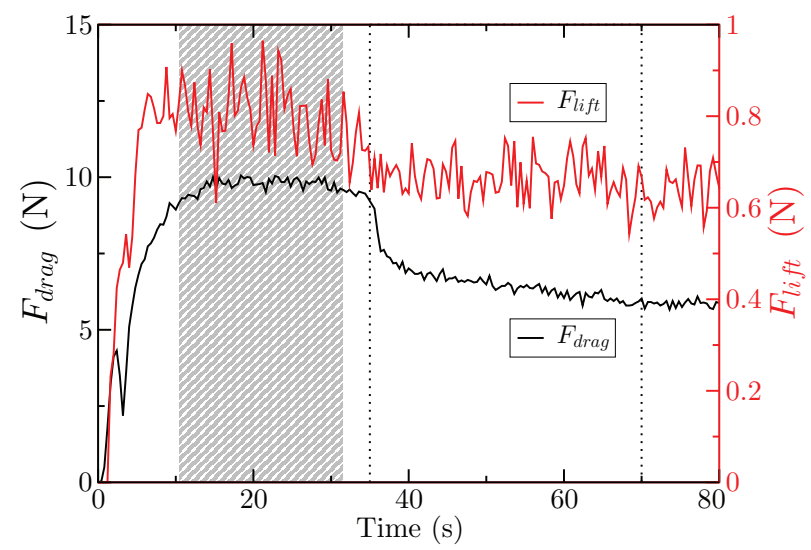

FIG. 2. Time evolution of the drag and lift forces on a cylinder $D=4 \mathrm{~mm}$ and $L=12 \mathrm{~cm}$, at depth $h=10.3 \mathrm{~cm}$ and angular velocity $\Omega=0.8 \mathrm{rpm}$. The gray region corresponds to the averaging window. Dotted lines are half rotations. 


\section{B. Experimental results}

Figs. 3(a) and 3(b) show the drag and lift forces measured on three cylinders having the same diameter $D=4 \mathrm{~mm}$ but different length $(L=6,9$, and $12 \mathrm{~cm})$. As observed in several previous studies, ${ }^{17,19}$ the drag force is proportional to the surface and to the depth $h$ of the object, confirming that no Janssen effect occurs in our configuration. In Fig. 3(c) the drag force rescaled by the apparent surface $F_{\text {drag }} / D L$ is plotted as a function of the hydrostatic pressure $\rho g h$, with $\rho=1.5 \mathrm{~g} \mathrm{~cm}^{-3}$ the density of the granular medium ( $\rho=\phi \rho_{g}$ with $\phi \simeq 0.6$ the volume fraction of the medium). The collapse of the data on a line shows the following scaling for the drag force:

$$
F_{\text {drag }}=C_{d} \rho g h D L,
$$

with $C_{d} \sim 13$. It is interesting to note that although the scaling is intuitively given by a friction criterium (a force proportional to the ambient hydrostatic pressure times the surface) the coefficient of proportionality is large. Such a large amplification factor of the drag forces in granular media is recovered in various configurations and depends on the geometry of the system as well as on the packing fraction and frictional properties of the particles. ${ }^{8,18,29}$

The remarkable result concerns the lift force plotted in Fig. 3(b). First of all, it must be noted that the vertical force points upward (hence its name "lift"), which means that it tends to push the object outside the granular bed. Second, the lift force shows a very different behaviour than the drag force. Close to the free surface, the lift force increases with depth as observed in Ding et al. ${ }^{24}$ However, at larger depths, the lift saturates and reaches a constant value independent of the depth. This saturation value of the lift $F_{\text {lift }}^{\text {sat }}$ increases when increasing the length of the cylinder (Fig. 3(b)). Based on a dimensional argument, a scaling can be proposed for the lift force. Far from the free surface, the only source of asymmetry that can induce a lift force is the gravitational pressure gradient $\nabla p=\rho g$. As a result, one expects the lift force to scale like the buoyancy force : $\nabla p \times$ Volume $=\rho g \pi(D / 2)^{2} L$. In Fig. 3(d), we have plotted the ratio between the lift force and the buoyancy as a function of the depth. The data obtained for cylinders having different lengths collapse on a single curve.

To further investigate the robustness of this scaling law, we have carried out systematic experiments varying both the diameter and the length of the cylinder. Fig. 4 is a summary of all our results showing how the lift force rescaled by the buoyancy varies as a function of $h / D$, the depth over the cylinder diameter. For all cylinders, the saturation of the lift force at large depths is recovered. However, the normalisation by the buoyancy force is not sufficient to capture the whole dependency of the lift force on the diameter. Data for small cylinders deviate from the main trend, with a higher plateau value reached at a larger depth. In order to analyze the influence of the diameter $D$, we have plotted in Fig. 5 the plateau value of the lift force $F_{\text {lift }}^{\text {sat }}$ rescaled by the buoyancy as a function of $D / d$, the cylinder diameter rescaled by the grain diameter. A master curve is obtained, giving the
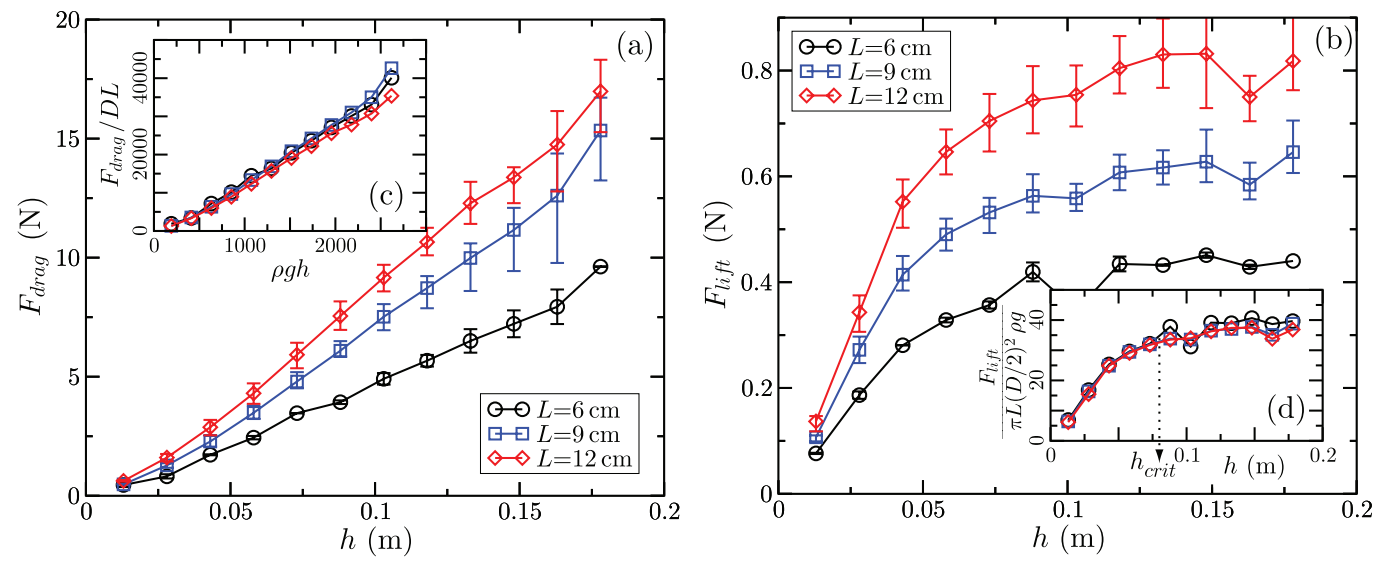

FIG. 3. Drag (a) and lift (b) forces as function of depth $h$ measured with cylinders of diameter $D=4 \mathrm{~mm}$ and various lengths $L$. Each point is an average of 4 measurements, error bars indicating their minimal and maximal values. (Inset) Scaled drag (c) and lift (d) forces for the same cylinders (see text). 


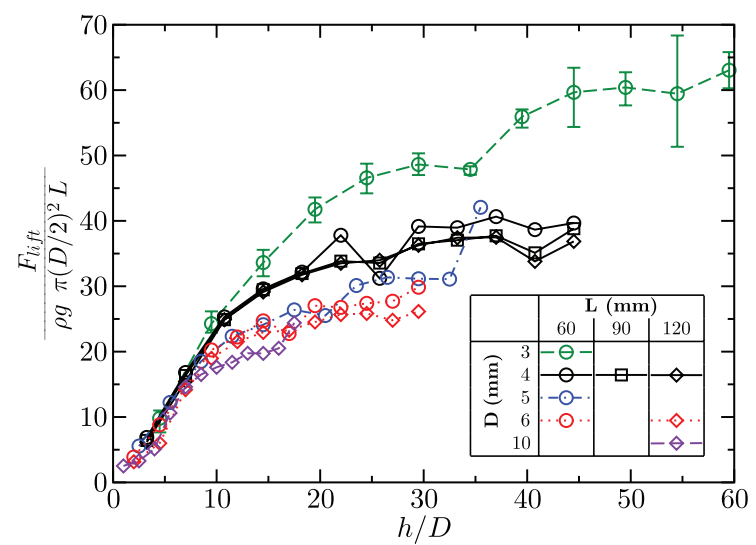

FIG. 4. Lift force rescaled by the buoyancy for cylinders of various lengths $L$ and diameters $D$ as function of the rescaled depth $h / D$. Each point is an average of 4 measurements, error bars indicating minimal and maximal measured values are displayed only for $D=3 \mathrm{~mm}$ for clarity.

following scaling of the saturated lift force as a function of the parameters:

$$
F_{\text {lift }}^{\text {sat }} \sim C_{l}(D / d) \rho g \pi \frac{D^{2}}{4} L .
$$

The factor $C_{l}(D / d)$ is a function of the ratio of the obstacle diameter relative to the grain diameter, and tends to a constant $C_{l} \approx 20$ when $D / d>15$. This means that for large obstacles compared to the grain diameter, the lift force is equal to 20 times the buoyancy force, a surprisingly high force. For smaller diameter this ratio increases and the lift force can reach values as high as 60 times the buoyancy for cylinders having a diameter less than 5 grain diameters. Such a finite size effect has been observed in previous studies for the drag force, which is found to be higher for small objects than for larger ones. ${ }^{13,17}$ We observe the same effect for the lift force.

This finite size effect is also observed when studying the critical depth $h_{\text {crit }}$ at which the lift force saturates to its plateau value (see Fig. 3(d)). Fig. 6 shows that for large cylinder diameters (typically $D / d>15$ ), the critical saturation depth $h_{\text {crit }}$ is independent of the grain size and scales as $h_{\text {crit }} \approx 10 D$. However, for cylinder diameter close to the grain diameter, the saturation depth increases strongly: typically, a 5 grain diameters cylinder needs to reach a depth equal to 40 times its diameter for the lift force to saturate.

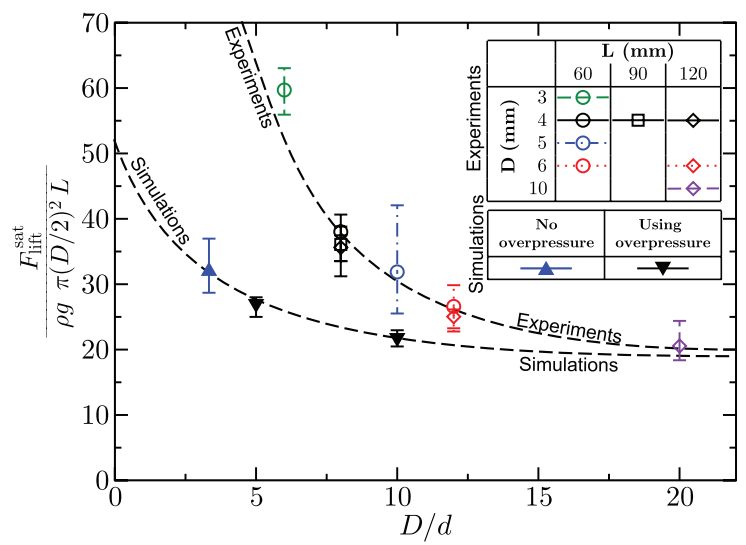

FIG. 5. Saturated lift force scaled by buoyancy as function of the ratio between the cylinder diameter to the grain diameter $D / d$, both in experiments and discrete simulations. Points are average of $F_{\text {lift }}$ in the saturated region, error bars are maximal and minimal $F_{\text {lift }}$ in this region. The lines are guides for the eyes. 


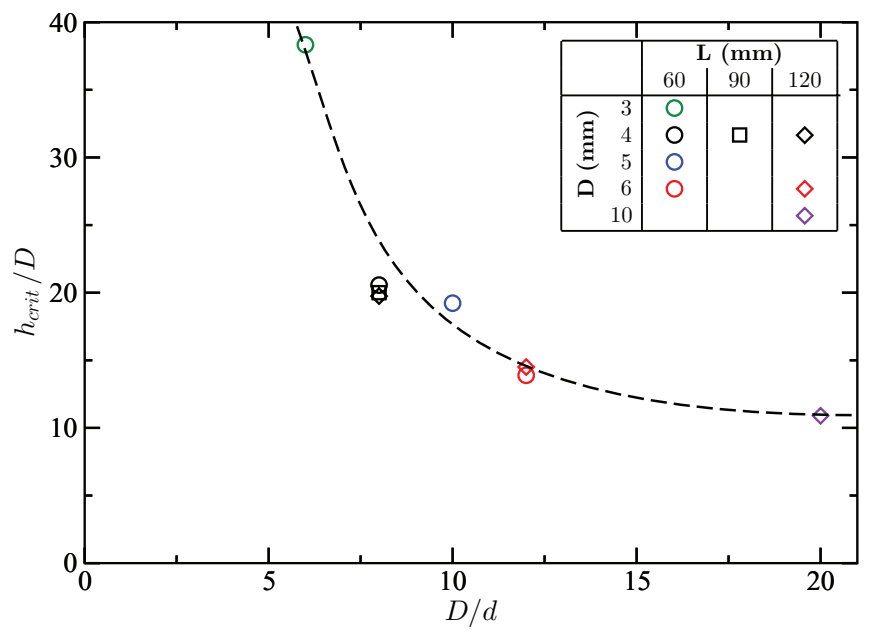

FIG. 6. Critical saturation depth scaled by the cylinder diameter $h_{\text {crit }} / D$ as function of $D / d$ in the experiments. The saturation depth $h_{\text {crit }}$ is defined as the depth at which the lift force reaches $90 \%$ of the plateau value (see Fig. 3(d)). The line is a guide for the eyes.

In conclusion to this experimental section, we have shown that a cylinder moving horizontally in a granular medium not only experiences a drag force, but also a very high lift force, despite the top/bottom symmetry of the object. Close to the free surface, the lift force increases with depth, as already observed in the experiment by Ding et al. ${ }^{24}$ However, at large depths, we found that the lift saturates and scales with the buoyancy force, its order of magnitude being equal to 20 times the buoyancy in our experiment with glass beads.

To better understand how this lift force arises from the stress distribution applied by the grains on the moving cylinder, and since it remains a challenge to probe experimentally the local stress distribution in a granular medium, we perform in Sec. III numerical simulations using a discrete element method.

\section{NUMERICAL SIMULATIONS}

\section{A. Numerical method}

To perform numerical simulations of a cylinder moving in a granular medium we have used the open source software LIGGGHTS..$^{30}$ The software is based on a molecular dynamics method and solves the equations of motion for the dynamics of soft, inelastic, frictional spheres. Details of the simulation method are given in Appendix A. The configuration studied is sketched in Fig. 7. The simulation space is a 3D box with periodic boundary conditions along the $x$ and $y$ directions. Gravity points towards $-z$. The size of the box is $140 \times 10 \times 120$ particles in the $x, y$, and $z$ direction. At the beginning of the simulations, a monolayer of fixed spheres is created at the bottom of the simulation box. Grains are then poured in the box, until the surface reaches the desired vertical position for the cylinder. A cylinder of diameter $D$ is then introduced with its axis aligned along the $y$ direction. The cylinder is treated as a fixed wall and remains static during the simulation. Once the cylinder has been created, more grains are poured in the box up to the desired level. To create the relative motion between the cylinder and the granular medium, a velocity $U_{0}$ is then prescribed to the bottom monolayer, which entrains the whole granular bed. Simulating the flow when the cylinder is deeply buried in the packing is expensive in term of CPU time, due to the large amount of grains above the cylinder. In order to simulate the flow at high pressure level without increasing the height of the packing and spending too much time, the following trick has been used. Before starting the motion, when the packing has been created, an additional square lattice monolayer of heavy grains of density $\rho_{g}^{*}$ higher than $\rho_{g}$ is placed at the top of the packing, with typically $60 \rho_{g}<\rho_{g}^{*}<210 \rho_{g}$. Those grains are free to move vertically but their horizontal velocity is imposed and equal to the bottom 


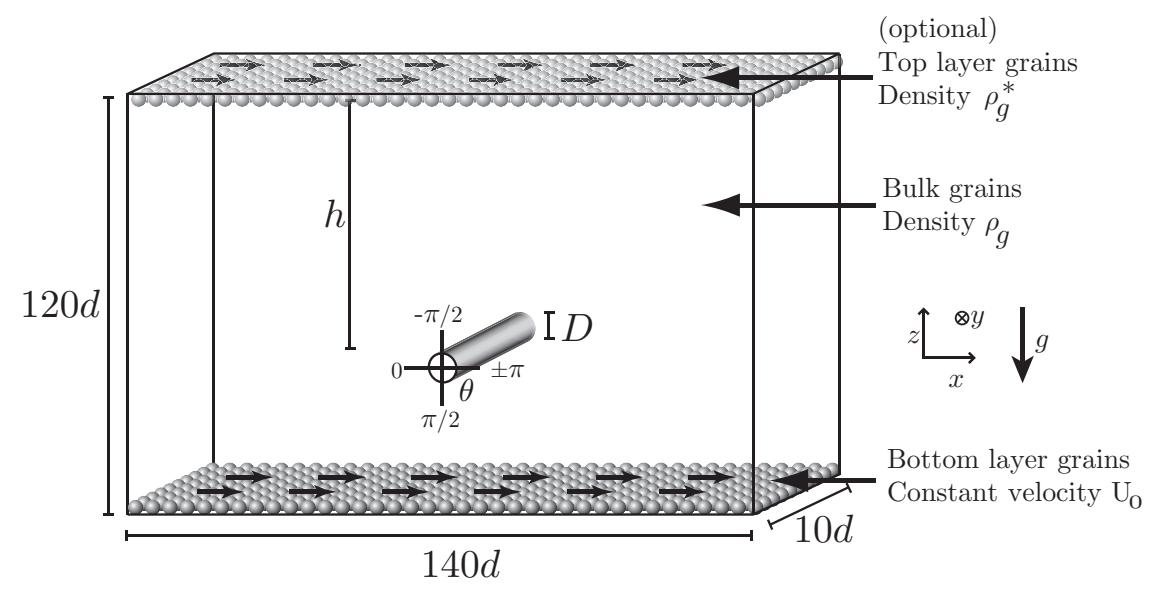

FIG. 7. Sketch of the molecular dynamic simulation geometry.

velocity $U_{0}$, which, far from the cylinder, induces a motion of the medium as a block. With this trick, the cylinder feels an ambient hydrostatic pressure corresponding to a column of equivalent height $\tilde{h}=h+\frac{\pi d}{6} \frac{\rho_{g}^{*}}{\rho}$. This procedure is used with $h / D=16$, corresponding at a cylinder at the center of the granular bed.

In this study, we have performed three kinds of numerical experiments. (i) The first one corresponds to the "normal" situation that mimics the experiments with no heavy grains at the top. This simulation was essentially used to study moderated pressure levels. In this case, the effective depth $\tilde{h}$ is simply the real distance $h$ from the centre of the cylinder to the free surface: $\tilde{h}=h$. (ii) The second kind of simulations was used to study high pressure levels by adding the top heavy monolayer, giving an equivalent $\tilde{h}=h+\frac{\pi d}{6} \frac{\rho_{s}^{*}}{\rho}$, with $h / D=16$. (iii) Finally, we have also performed simulations where gravity only applies on the top heavy monolayer but is switched off for the grains in the bulk. This latter case provides an interesting way to probe the flow in presence of a mean pressure equivalent to a column of equivalent height $\tilde{h}=\frac{\pi d}{6} \frac{\rho_{8}^{*}}{\rho}$ but without pressure gradient.

The typical time of each simulation corresponds to a relative displacement between the cylinder and the granular medium equal to a 40 cylinder diameters (a typical run cost $4.5 \times 10^{5} \mathrm{~s}$ total CPU time, meaning $15 \mathrm{~h}$ in real time on the parallel computer from IDRIS). A steady regime is typically reached after the cylinder has moved 2 diameters. All the quantities presented in the following are time averaged in the steady regime.

\section{B. Averaged drag and lift forces}

Intensive simulations have been carried out for different cylinder diameters and different equivalent depths. We first report results for the average forces experienced by the obstacle. Fig. 8 shows how the drag and the lift forces vary with depth in the simulations. The same behaviours as in the experiments are observed. Fig. 8(a) shows that the ratio of the drag force over the surface $F_{\text {drag }} / D L$ varies linearly with the effective hydrostatic pressure $\rho g \tilde{h}$. The coefficient of proportionality between the rescaled drag force and the hydrostatic pressure in the simulations $\left(C_{d} \sim 10\right)$ is also similar as in the experiments. It is interesting to notice that no difference is observed between the case with and without pressure gradient, indicating that the drag is mostly controlled by the mean pressure level. The simulations of the lift force also reproduce well the results observed in experiments. The lift force first increases and saturates at equivalent depths large compared to the cylinder diameter. The interesting point is that, unlike the drag force, the presence or not of a pressure gradient has a strong influence on the lift force: no significative lift is obtained for the case without pressure gradient, indicating that the origin of the lift is the pressure gradient.

Quantitatively, the lift force measured in the simulations is close to the experimental data for large cylinders, but a systematic deviation is observed for small diameters as shown in Fig. 5. 

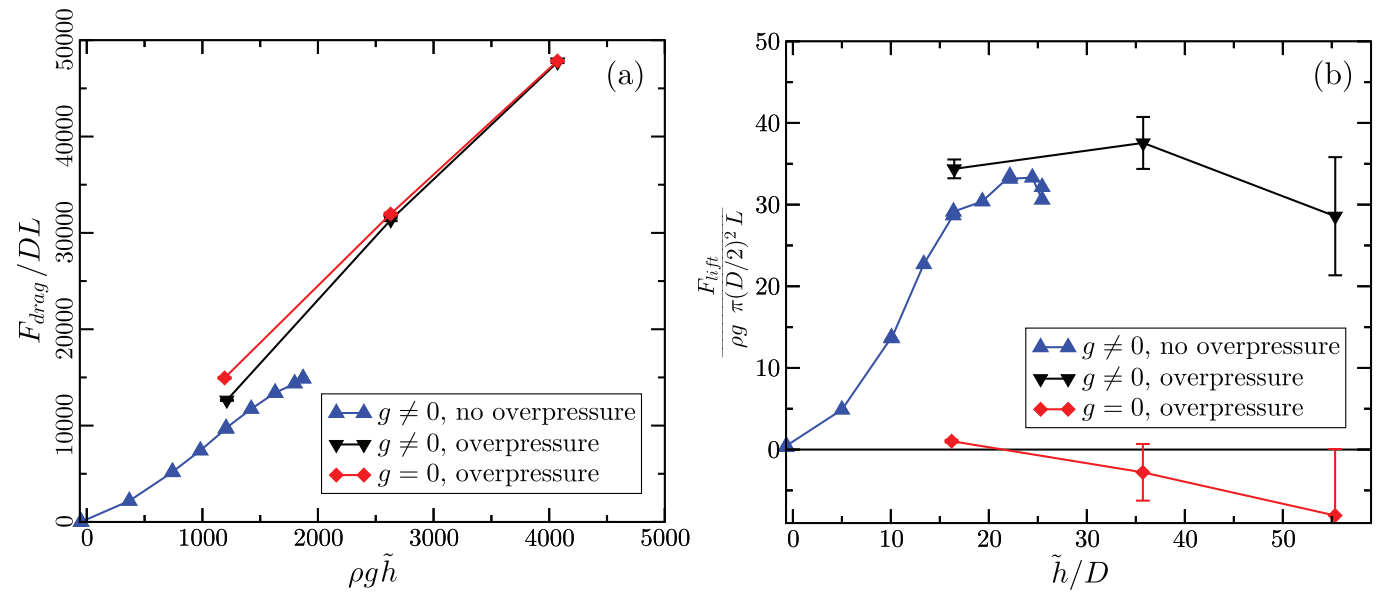

FIG. 8. Simulated drag (a) and lift (b) forces measured for a cylinder $D / d=3.3$ using the 3 different ways of changing the effective depth $\tilde{h}$. Error bars are standard deviation on 4 runs.

The lift rescaled by the buoyancy force is always $20 \%$ to $50 \%$ less in the simulations than in the experiments. The discrepancies may be attributed to the difference in the geometry (a rotating device in the experiment versus a translating object in the simulation), to the choice of the contact model, or to slight differences in the initial state of the packing (volume fraction, polydispersity). However, qualitatively, all the features observed experimentally are recovered in the simulations showing that the main feature, i.e. the saturation of the lift force with depth, is a robust effect.

\section{Flow around the obstacle}

To understand the origin of the lift force and its saturation observed at large depth, we first analyze the properties of the flow around the obstacle. The discrete simulations give access to the particle velocities and to the inter-particle contact forces, from which the continuous velocity field and the stress field can be computed using a coarse graining procedure described in Appendix A. Fig. 9 presents successively the pressure field $(a, b)$, the velocity field in the frame moving with the obstacle (c,d), and the velocity field in the frame moving with the particles far away from the obstacle (e,f). For each case, we compare the normal configuration where the obstacle moves in the presence of the gravity field (right), with a case where the obstacle experiences the same effective mean pressure but without gravity, i.e. without any pressure gradient (left). From these figures, the crucial role of the pressure gradient in the development of a lift force is evident. Without pressure gradient, the flow and pressure fields exhibit a top/bottom symmetry as expected. When the pressure gradient is switch on, the flow and pressure fields become asymmetric.

We first analyse the pressure field. In absence of gravity, a high pressure region develops in front of the obstacle and a low pressure region at the back (Fig. 9(a)). When gravity is switch on (Fig. 9(b)), the pressure field is deformed and the high and low pressure zones are no longer aligned with the central horizontal line. The high pressure region is slightly tilted downward and the low pressure zone at the rear is tilted upward. The velocity field is also affected by the pressure gradient. Comparing Figs. 9(c) and 9(d), one observes that when a pressure gradient exists, particles are more inclined to pass above the obstacle (the central line is deformed upward) and move faster above than below compared to the symmetric case. When looking at the velocity field in the frame moving with the grains far away from the cylinder (Figs. 9(e) and 9(f)), one observes that the shape of the zone affected by the moving obstacle becomes asymmetric in presence of gravity, with a larger extension above than below the cylinder. The role of the pressure gradient thus appears to be more subtile than simply adding a linear vertical variation of the stress, as it considerably affects the flow around the cylinder. 

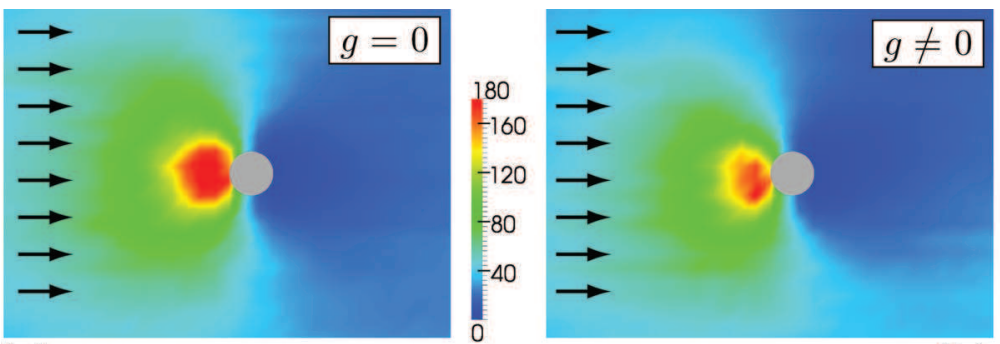

(a) $P / \rho_{g} g d$

(b)
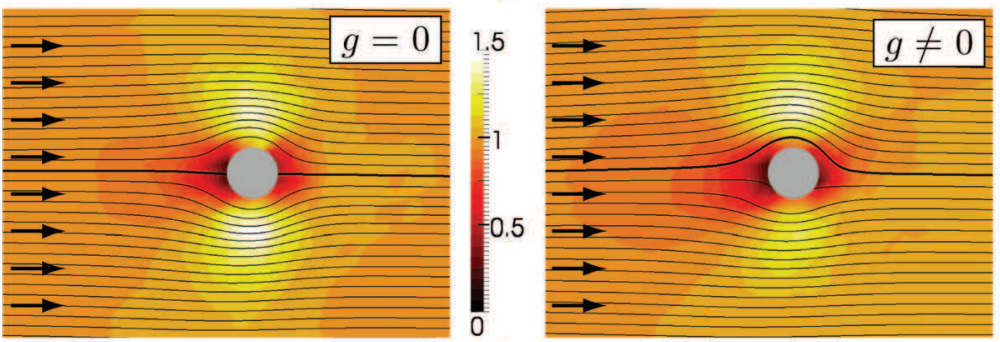

(c)

$\|U\| / U_{\mathrm{o}}$

(d)

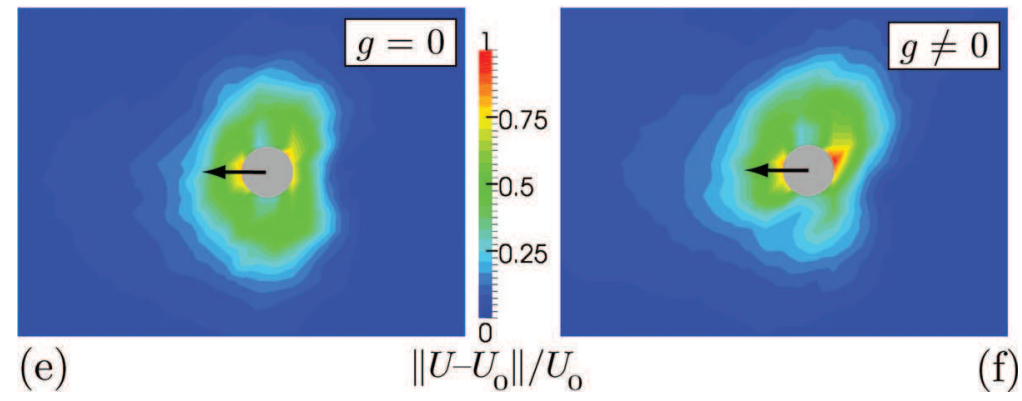

FIG. 9. Discrete numerical simulations for a cylinder of diameter $D / d=3.3$ located at an effective depth $\tilde{h} / D=16$. Figures on the left (resp. on the right) correspond to simulations without (resp. with) pressure gradient. (a,b) Pressure field. (c,d) Velocity field in the frame of the cylinder showing the magnitude of the velocity and the streamlines. (e,f) Velocity field in the frame of the grains far away from the cylinder.

\section{DISCUSSION}

Both the experiments and the simulations presented above show that a cylinder moving at large depth in a granular medium under gravity experiences a strong lift force, which is independent of the depth and scales like the buoyancy. The striking result is that this lift force is equal to more than 20 times the common Archimedes force in fluid mechanics. To our knowledge, such a lift force induced by motion on a symmetrical object is peculiar to granular materials and has no equivalence in newtonian or non-newtonian fluids.

To understand the origin of this lift, it is interesting to compare how the efforts are distributed at the surface of the obstacle in the case without and with gravity (i.e., without or with pressure gradient). Fig. 10 is a qualitative picture of the scenario. When a cylinder moves in a granular medium under a mean pressure $P_{0}$ but in absence of pressure gradient (no gravity), a strong asymmetry exists between the front and the rear as observed in Fig. 9(a), with a very large overpressure about 15 times the mean pressure level at the front. In this case both the top and bottom half of the cylinder are submitted to strong but opposite net vertical forces, resulting in no net lift force on the cylinder. When a pressure gradient is present, one expects from the frictional rheology of granular media that the stress on the cylinder scales with the pressure level. Since the top and bottom parts feel a different hydrostatic pressure due to gravity, the pressure on the lower front quarter should be larger 

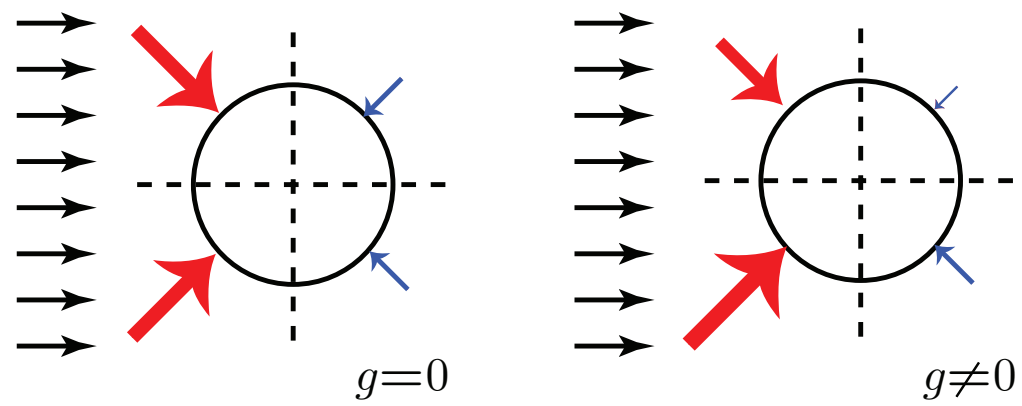

FIG. 10. Schematic forces on each quarter of a cylinder in a granular flow, either with or without pressure gradient.

than on the upper front quarter, inducing a net lift force. In this picture, the lift force comes from two factors: (i) the large overpressure that develops at the front of an object moving in a granular medium (left/right asymmetry) and (ii) the top/bottom asymmetry induced by the gravity.

In order to more precisely test this qualitative argument, we have computed the stress distribution on the cylinder from the discrete forces applied by the grains in contact with the cylinder (see Appendix A). Fig. 11(a) shows the radial distribution for both the normal stress $\sigma_{n}$ (thick black line) and the tangential stress $\sigma_{t}$ (thin gray line) for a case with gravity (solid line) and without gravity (dotted line). The first important observation is that the tangential stress is always one order of magnitude smaller than the normal stress and thus contributes to less than $10 \%$ of the total force experienced by the cylinder. In the following discussion, we will thus focus only on the normal stress distribution $\sigma_{n}(\theta)$ around the obstacle.

When gravity is zero and no pressure gradient exists, the normal stress distribution is symmetric around the center line of the cylinder, with a maximum at the front $(\theta=0)$ equal to 15 times the confining pressure $P_{0}$ and an almost vanishing value at the rear $(\theta=\pi)$ (Fig. 11(c)). By increasing the confining pressure (i.e., the equivalent depth) (Figs. 11(b) and 11(e)), one observes that this symmetric distribution increases linearly with the confining pressure, as expected from the frictional rheology. One can then write $\sigma_{n}^{g=0}(\theta)=\rho g \tilde{h} \tilde{\sigma}_{n}^{g=0}(\theta)=P_{0} \tilde{\sigma}_{n}^{g=0}(\theta)$ with the normalised distribution $\tilde{\sigma}_{n}^{g=0}(\theta)$ being independent of $P_{0}$ (Fig. 11(d)).

When gravity is switched on, the distributions become asymmetric (Fig. 11(b)): the normal stress becomes higher in the bottom front quarter $(0<\theta<\pi / 2)$ and lower in the top front quarter $(-\pi / 2<\theta<0)$. This asymmetry induces the net lift force. Qualitatively, the simple picture of Fig. 10 is then correct: the lift arises from an increase of the normal stress on the bottom front quarter. One can wonder if this asymmetry of the stress distribution in the presence of gravity is
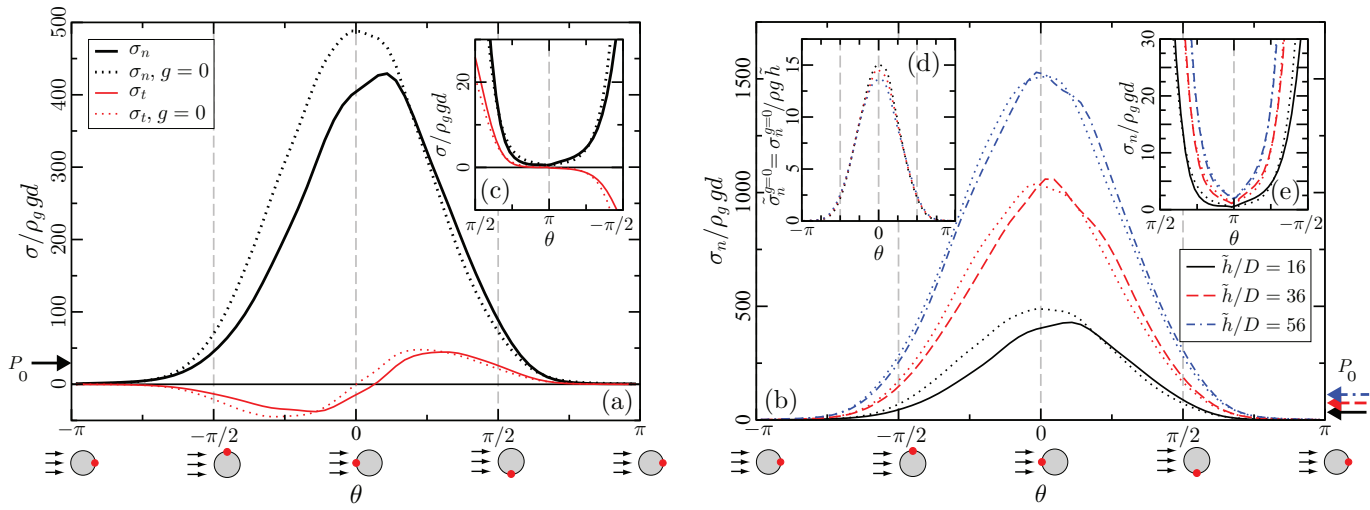

FIG. 11. (a) Normal and tangential stress distribution for a cylinder $D / d=3.3$ and $\tilde{h} / D=16$. (b) Normal stress distribution at various effective depth around a cylinder $D / d=3.3$. Dotted lines are with $g=0$ (no pressure gradient). Insets: (c) and (e) magnification of the rear part of the cylinder; (d) normal stress scaled by the hydrostatic pressure. 
simply given by the stress distribution without gravity, weighted by the hydrostatic pressure level. In other terms, it is tempting to assume that the forces on each elementary surface around the obstacle only depends on the local hydrostatic pressure and is the same with or without pressure gradient. Under this assumption, the normal stress distribution $\sigma_{n}(\theta)$ around the obstacle in presence of gravity would be given by the normalised stress distribution without gravity $\tilde{\sigma}_{n}^{g=0}(\theta)$ multiplied by the local hydrostatic pressure as

$$
\sigma_{n}(\theta)=\tilde{\sigma}_{n}^{g=0}(\theta) \rho g\left(\tilde{h}+\frac{D}{2} \sin \theta\right),
$$

where $\tilde{h}$ is the equivalent depth at the centre of the cylinder. In this case, the lift force would be simply given by the integral $F_{\text {lift }}=\left(\rho g L D^{2} / 4\right) \int_{-\pi}^{\pi} \tilde{\sigma}_{n}^{g=0}(\theta) \sin ^{2} \theta d \theta$ since $\int_{-\pi}^{\pi} \tilde{\sigma}_{n}^{g=0}(\theta) \sin \theta d \theta=0$. This approach thus predicts that the lift scales like the buoyancy, in agreement with the experiments and simulations. However, this simple description fails in quantitatively predicting the lift force. Using the distribution $\tilde{\sigma}_{n}^{g=0}$ measured in the simulation without gravity (Fig. 11(d)), one find $F_{\text {lift }} /\left(\pi \rho g L D^{2} / 4\right) \simeq 3.6$, which is one order of magnitude lower than the measured lift force, equal to 30 times the buoyancy in this case. The discrepancy can also be evidenced by directly looking at the asymmetric part of the distribution. Fig. 12 shows the difference $\sigma_{n}-\sigma_{n}^{g=0}$ normalized by $\rho g D$ for the three equivalent depths of Fig. 11(b). As in the simple model, the three curves roughly collapse, showing that the asymmetric part of the distribution does not scale with the mean pressure. However, the asymmetric contribution predicted by Eq. (4) and shown by the dotted line in Fig. 12 is one order of magnitude smaller than the measured ones.

The important result of this analysis is then that the angular stress distribution on the obstacle is the sum of a symmetric part proportional to the mean pressure or equivalently to the depth, which is responsible for the drag force but do not contribute to the lift, and a asymmetric part which does not depends on the mean pressure and induces lift. However, the asymmetry in the stress distribution cannot be understood by a simple weighting of the stress by the local hydrostatic pressure, but results from a more complex modification of the whole flow around the obstacle induced by the pressure gradient. It is interesting to note that the splitting of the stress between a symmetric part proportional to depth and an asymmetric part independent of depth can be rationalised in an asymptotic analysis presented in Appendix B. In this analysis, the small parameter is $\epsilon=\rho g D / P_{0}=D / \tilde{h}$, the typical pressure variation on the size of the cylinder over the mean pressure, and one assumes that the rheology is given by a frictional criterium.

Before concluding, it is interesting to discuss our observations in the light of the results previously obtained by Ding et al. ${ }^{24}$ A first result in their study is that the lift force varies linearly with

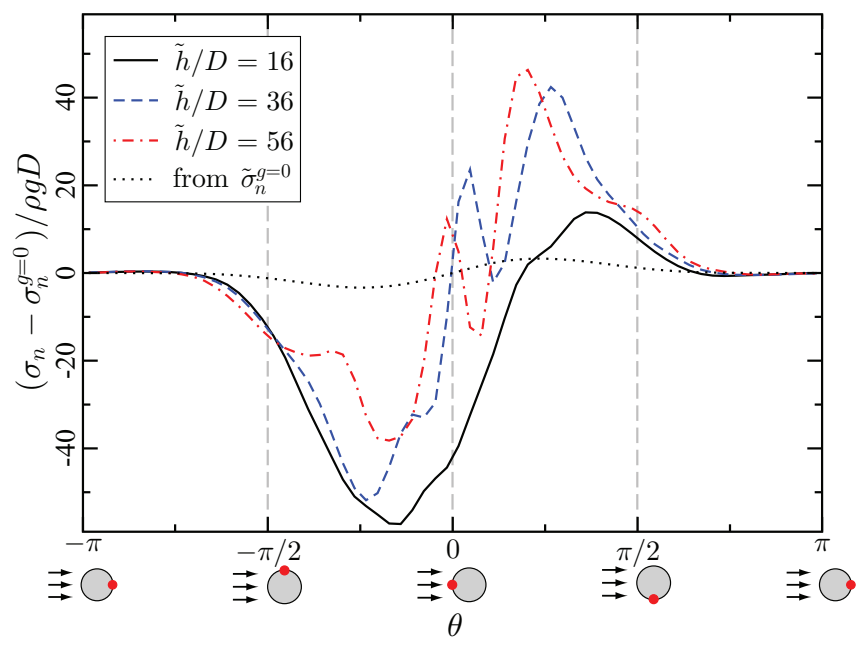

FIG. 12. Angular distribution of the difference between pressure with and without bulk gravity $\sigma_{n}-\sigma_{n}^{g=0}$ for 3 effective depths. 


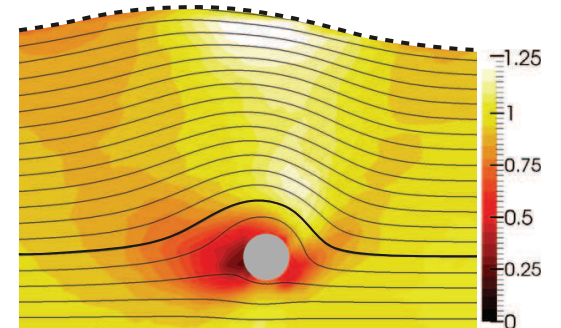

(a)
$\|U\| / U_{0}$

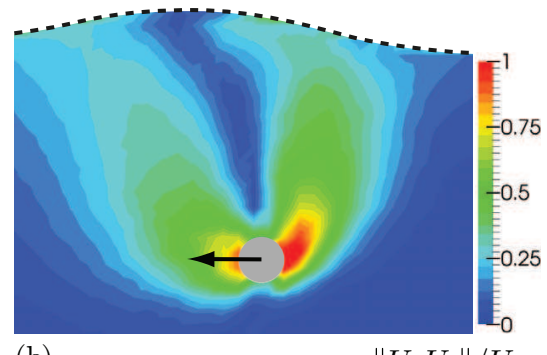

(b)
$\left\|U-U_{0}\right\| / U_{0}$

FIG. 13. Discrete numerical simulation for a cylinder of diameter $D / d=3.3$ located at a depth $h / D=\tilde{h} / D=5$, without overpressure. The dashed black line indicates the free surface. (a) Velocity field in the frame of the cylinder showing the magnitude of the velocity and the streamlines. (b) Velocity field in the frame of the grains far away from the cylinder.

the depth like the drag force. Our experiments show that this is only true when the object moves close to the free surface, at a distance less than 15 cylinder diameters, and that the lift saturates at larger depth. The linear increase of the lift is thus a surface effect, as shown in Fig. 13, where it becomes clear that at low depth the perturbed zone around the cylinder reaches the free surface and deform it. The second result of Ding et al. ${ }^{24}$ is a phenomenological model to compute the lift force on objects of arbitrary shape from the knowledge of the force on plates at various inclinations. The basic assumption in the model is that each surface element of the obstacle experiences the same lift force as a plate having the same angle of attack and weighted by the local hydrostatic pressure. This model predicts the drag and lift forces close to the surface for complex objects and has been applied with success to describe legged locomotion in the framework of resistive force theory. ${ }^{27}$ However, if we use data of Ding et al. ${ }^{24}$ for the forces on plates in our case, the saturation of the lift force at large depths is not recovered. One may argue that the measurement on plates was done close to the free surface and that measurements at larger depths may reconcile both regimes. However, the fact that, in our study, the lift force cannot be deduced from the simple multiplication of the stress distribution obtained without gravity by the local hydrostatic pressure suggests a more complex picture. The presence of the pressure gradient at the origin of the lift actually perturbs the flow at the scale of the whole object, which precludes the estimate of the lift just from local measurements on plates. Understanding why the resistive force theory works well in shallow condition and fails for deeply buried objects remains an open issue.

\section{CONCLUSION}

In conclusion, we have shown that a cylinder moving in a granular bed experiences a strong lift force, which saturates at large depths. The lift far from the free surface is proportional to the buoyancy of the object, but with a high amplification factor, higher than 20. The origin of this strong lift force has been studied using discrete numerical simulations and shown to arise from the gravitational pressure gradient, which breaks the up/down symmetry. However, the analyse of the stress distribution around the object reveals that the lift cannot be understood by a simple weighting of the stress by the local hydrostatic pressure, but results from a more complex modification of the flow around the obstacle. These results could have implications for models describing the forces acting on objects buried in granular media such as in animals and robots locomotion. ${ }^{26,27}$

Our study also open new perspectives for the basic understanding of granular flows. First, it would be interesting to address the question of drag and lift forces in granular media in the framework of continuum modeling. We have seen that the existence of a strong lift force is specific to granular media and related to the frictional behaviour of these systems. The data presented here could serve as a base to test the relevance of rheological models proposed in the literature, in the spirit of recent studies who implemented viscoplastic rheologies ${ }^{31}$ in numerical codes. ${ }^{32-34}$ Another interesting perspective would be to extend the study of forces in granular media to objects immersed in granular sheared flows and not only in static piles. In fluid mechanics, it is well known that gradients of shear 
may induce net forces on particles, a question that would be relevant in the context of granular media and the problem of the segregation of large objects. ${ }^{35,36}$

\section{ACKNOWLEDGMENTS}

We acknowledge Marc Médale for fruitful discussions. This work has been supported by the French National Research Agency (ANR) through the program ANR Blanc RSC ANR-08-BLAN0048-CSD2 and by the Labex MEC ANR-11-LABX-0092 and A*MIDEX ANR-11-IDEX-000102. The numerical simulations have been performed using the HPC resources of IDRIS under the allocation 2012-96212 made by GENCI (Grand Equipement National de Calcul Intensif).

\section{APPENDIX A: SIMULATION METHOD}

The contact model used in the DEM simulations is a Hertzian model with friction and dissipation, ${ }^{30,37,38}$ but with no rolling friction nor cohesion. More precisely, for two particles of diameter $d$ and mass $m$ separated by a distance $r$, one defines the normal direction as the axe joining the centre of the particles, the tangential direction being in the plane perpendicular to that direction. The force is then zero when $r>d$. For $r<d$, one can define the normal overlap $\delta_{n}=d-r$, and the tangential overlap $\delta_{t}$ which is the relative tangential displacement of the particles since the beginning of the contact. The force is then decomposed in its normal part $F_{n}$ and the tangential part $F_{t}$ :

$$
\begin{aligned}
F_{n} & =k_{n} \delta_{n}+\gamma_{n} v_{n}^{r e l} & & \\
F_{t} & =k_{t} \delta_{t}+\gamma_{t} v_{t}^{r e l} & & \text { if }\left|F_{t}\right|<\mu_{g}\left|F_{n}\right| \\
& =\mu_{g}\left|F_{n}\right| & & \text { else }
\end{aligned}
$$

in which $v_{n}^{r e l}$ and $v_{t}^{r e l}$ are the normal and tangential components of the relative velocity of the particles at the contact points. The constant $\mu_{g}$ is the friction coefficient and the parameters $k_{n}, k_{t}, \gamma_{n}, \gamma_{t}$ are function of the material properties and of the overlaps $\delta_{n}$ and $\delta_{t}$ as given in Table I. Note that the coefficients corresponding to particle-particle contacts are slightly different than particle-cylinder contact, the last one being treated as a contact between a sphere and an infinite plane.

The particles used in the simulation are $d=1.5 \mathrm{~mm}$ in diameter, with density equal to $\rho_{g}=2.5 \mathrm{~g} \mathrm{~cm}^{-3}$. The contact interaction between particles and between the particles and the obstacle are characterized by a Young modulus equal to $E=5 \times 10^{6} \mathrm{~Pa}$, a Poisson ratio of $v=0.45$, a coefficient of restitution $e=0.6$, and a coefficient of friction $\mu_{g}=0.5$. These values are classical values used in MD simulations to model the flow of glass beads. ${ }^{39}$ Our choice to use dimensional quantities instead of dimensionless variables in the simulations is motivated by the fact that we have performed simulations both with and without gravity. The two cases corresponding to different pressure scales, it turns out to be easier from a simulation point of view to use dimensional quantities and use dimensionless quantities afterwards when analyzing the results.

\begin{tabular}{|c|c|c|c|c|}
\hline Coefficient & $\begin{array}{l}\text { Particle-particle } \\
\text { expression }\end{array}$ & Value & $\begin{array}{l}\text { Particle-cylinder } \\
\text { expression }\end{array}$ & Value \\
\hline$k_{n} / \sqrt{\delta_{n}}=k_{n}^{*}$ & $\frac{E}{3\left(1-v^{2}\right)} \sqrt{d}$ & $81 \times 10^{3} \mathrm{~N} \mathrm{~m}^{-\frac{3}{2}}$ & $\frac{2 E}{3\left(1-v^{2}\right)} \sqrt{d / 2}$ & $114 \times 10^{3} \mathrm{~N} \mathrm{~m}^{-\frac{3}{2}}$ \\
\hline$k_{t} / \sqrt{\delta_{n}}=k_{t}^{*}$ & $3 \frac{1-v}{2-v} k_{n}^{*}$ & $86 \times 10^{3} \mathrm{~N} \mathrm{~m}^{-\frac{3}{2}}$ & $3 \frac{1-v}{2-v} k_{n}^{*}$ & $122 \times 10^{3} \mathrm{~N} \mathrm{~m}^{-\frac{3}{2}}$ \\
\hline$\gamma_{n} / \delta_{n}^{1 / 4}$ & $-\sqrt{\frac{5}{2}} \beta \sqrt{m k_{n}^{*}}$ & $0.15 \mathrm{~kg} \mathrm{~s}^{-1} \mathrm{~m}^{-\frac{1}{4}}$ & $-\sqrt{5} \beta \sqrt{\sqrt{2} m k_{n}^{*}}$ & $0.26 \mathrm{~kg} \mathrm{~s}^{-1} \mathrm{~m}^{-\frac{1}{4}}$ \\
\hline$\gamma_{t} / \delta_{t}^{1 / 4}$ & $-\sqrt{\frac{5}{3}} \beta \sqrt{m k_{t}^{*}}$ & $0.13 \mathrm{~kg} \mathrm{~s}^{-1} \mathrm{~m}^{-\frac{1}{4}}$ & $-\sqrt{\frac{10}{3}} \beta \sqrt{\sqrt{2} m k_{t}^{*}}$ & $0.21 \mathrm{~kg} \mathrm{~s}^{-1} \mathrm{~m}^{-\frac{1}{4}}$ \\
\hline
\end{tabular}

TABLE I. Expressions and values of the coefficients of the contact force model used in the simulation. Here $\beta=\frac{\ln (e)}{\sqrt{\ln ^{2}(e)+\pi^{2}}}$. 
Using this contact force model, the time evolution of the motion of the particles is integrated using a Verlet algorithm with a constant time-step. The typical time-step is $10^{-5} \mathrm{~s}$.

\section{Coarse-graining method}

\section{a. Velocity field}

The continuum velocity field is obtained from the discrete positions $\mathbf{x}_{i}$ and velocities $\mathbf{v}_{i}$ of all the $N_{p}$ particles using the coarse-graining method developed by Glasser et al. ${ }^{10,40}$ The idea is to do a spatial averaging around points in the simulation space. The typical grid of points we use is 120 $\times 3 \times 120$ points in the simulation space. The averaging window is a sphere of radius $R$ centered at point $\mathbf{x}$. The mean velocity is then given by

$$
\mathbf{v}(\mathbf{x})=\frac{\sum_{i=1}^{N_{p}} \mathbf{v}_{i} \times \mathcal{W}\left(\mathbf{x}_{i}, \mathbf{x}\right)}{\sum_{i=1}^{N_{p}} \mathcal{W}\left(\mathbf{x}_{i}, \mathbf{x}\right)}
$$

where $\mathcal{W}\left(\mathbf{x}_{i}, \mathbf{x}\right)$ is the averaging function: $\mathcal{W}\left(\left|\mathbf{x}_{i}-\mathbf{x}\right|<R-d / 2\right)=1 / 6 \pi d^{3}$ and $\mathcal{W}\left(\left|\mathbf{x}_{i}-\mathbf{x}\right|\right.$ $>R+d / 2)=0$. In the simulations we use $R=d$.

\section{b. Stress field}

The stress tensor is computed using the same procedure from the forces $\mathbf{f}_{i j}$ between particles and is given by

$$
\boldsymbol{\sigma}(\mathbf{x})=\frac{1}{2} \sum_{i, j, i \neq j}^{N_{p}, N_{p}} \mathbf{f}_{i j} \otimes \mathbf{x}_{i j} \mathcal{W}\left(\mathbf{x}_{i j}, \mathbf{x}\right),
$$

where $\mathbf{x}_{i j}=\mathbf{x}_{j}-\mathbf{x}_{i}$ and $\mathcal{W}$ is the fraction of the contact vector $\mathbf{x}_{i j}$ inside the averaging region. ${ }^{10,40}$ Note that the kinetic part of the stress tensor is negligible in the quasi-static regime studied here. These fields are then averaged in time.

\section{c. Angular distribution of the stress on the cylinder}

To compute the stress on the cylinder, we extract for all the $N_{p c}$ contacts particle-cylinder, the radial position of the contact points $\theta_{i}$ and the force $\mathbf{f}_{\mathbf{i}}$ at the contact. The perimeter is divided into $M$ points at angles $\theta_{m}=(m+1 / 2) \times 2 \pi / M, m \in\{0 . . M-1\}$. The mean force $\mathbf{f}\left(\theta_{m}\right)$ at point $m$ is the average of the contact forces weighted by a gaussian function of mean $\theta_{m}$ and standard deviation $\varsigma$. This force is also averaged on the $y$ direction and temporally:

$$
\mathbf{f}\left(\theta_{m}\right)=\sum_{i=1}^{N_{p c}} \frac{\sqrt{2 \pi}}{M \varsigma} \exp \left(-\frac{\left(\theta_{i}-\theta_{m}\right)^{2}}{2 \varsigma^{2}}\right) \mathbf{f}_{i} .
$$

We typically used $\varsigma=8^{\circ}$ and $M=64$. The normal and tangential stresses $\sigma_{n}$ and $\sigma_{t}$ are given by: $\sigma_{n}\left(\theta_{m}\right)=\frac{\mathbf{f}\left(\theta_{m}\right) \cdot \mathbf{n}}{L D \pi / M}$ and $\sigma_{t}\left(\theta_{m}\right)=\frac{\left|\mathbf{f}-\left(\mathbf{f}\left(\theta_{m}\right) \cdot \mathbf{n}\right) \mathbf{n}\right|}{L D \pi / M}$ with $\mathbf{n}=\left(\cos \theta_{m}, \sin \theta_{m}\right)$.

\section{APPENDIX B: ASYMPTOTIC ANALYSIS}

We show in this appendix that the stress distribution around a cylinder immersed at large depth in a granular medium can be split between a symmetric part proportional to the pressure and an asymmetric part proportional to the gradient of pressure. To this end, we denote $D$ the diameter of the cylinder, $h$ the depth and $P_{0}=\rho g h$ the mean hydrostatic pressure at the centre of the cylinder. At large depth, the parameter $\epsilon=\frac{\rho g D}{P_{0}}=D / h$, which compares the pressure variation on the size of the cylinder to the confining pressure, is small $\epsilon \ll 1$. We then assume that the rheology of the granular medium is given by a friction criterium, i.e., the stress tensor $\sigma$ can be written as $\sigma=P(-\mathbf{I}+\boldsymbol{\mu})$, where $P$ is the pressure (the trace of the stress tensor) and $P \boldsymbol{\mu}$ is the deviatoric part. When there is no pressure gradient but a mean confining pressure $P_{0}$, i.e., $\epsilon=0$, the stress tensor can be written as 
$P=P_{0}\left(1+f_{0}(\mathbf{x})\right)$ and $\boldsymbol{\mu}=\boldsymbol{\mu}_{0}(\mathbf{x})$, where $f_{0}(\mathbf{x})$ and $\boldsymbol{\mu}_{0}(\mathbf{x})$ vanish far away from the cylinder and are symmetric under the transformation $z \rightarrow-z, z$ being the vertical coordinate with the origin at the centre of the cylinder. When switching on the gravity, one can write at first order in $\epsilon$ :

$$
P=P_{0}\left(1+\epsilon \frac{z}{D}+f_{0}(\mathbf{x})+\epsilon f_{1}(\mathbf{x})\right)
$$

and

$$
\boldsymbol{\mu}=\mu_{0}(\mathbf{x})+\epsilon \mu_{1}(\mathbf{x})
$$

where $f_{1}(\mathbf{x})$ and $\boldsymbol{\mu}_{1}(\mathbf{x})$ vanish at infinity. At first order, the stress tensor is given by

$$
\sigma=\tilde{\boldsymbol{\sigma}}_{0}+\epsilon \tilde{\boldsymbol{\sigma}}_{1}
$$

with

$$
\tilde{\boldsymbol{\sigma}_{0}}=P_{0}\left(1+f_{0}\right)\left(-\mathbf{I}+\boldsymbol{\mu}_{0}\right)
$$

and

$$
\tilde{\boldsymbol{\sigma}}_{1}=P_{0}\left[\left(\frac{z}{D}+f_{1}\right)\left(-\mathbf{I}+\boldsymbol{\mu}_{0}\right)+\left(1+f_{0}\right) \boldsymbol{\mu}_{1}\right] .
$$

The function $\tilde{\boldsymbol{\sigma}}_{0}$ is symmetric under the transformation $z \rightarrow-z$ whereas $\tilde{\boldsymbol{\sigma}}_{1}$ is a priori asymmetric. The only part that can induce lift thus comes from the second asymmetric term in the stress (the first order term in $\epsilon$ ), which scales like $\epsilon P_{0}=\rho g D$ and is thus independent of depth. This simple argument show that the lift at large depth scales like the buoyancy.

${ }^{1}$ H. Tabuteau, P. Coussot, and J. R. de Bruyn, "Drag force on a sphere in steady motion through a yield-stress fluid," J. Rheol. 51, 125-137 (2007).

${ }^{2}$ D. F. James, "Boger fluids," Annu. Rev. Fluid Mech. 41, 129-142 (2009).

${ }^{3}$ N. P. Chafe and J. R. de Bruyn, "Drag and relaxation in a bentonite clay suspension," J. Non-Newton. Fluid Mech. 131, 44-52 (2005).

${ }^{4}$ J. R. Gladden and A. Belmonte, "Motion of a viscoelastic micellar fluid around a cylinder: flow and fracture," Phys. Rev. Lett. 98, 224501 (2007).

${ }^{5}$ B. Dollet, F. Elias, C. Quilliet, C. Raufaste, M. Aubouy, and F. Graner, "Two-dimensional flow of foam around an obstacle: Force measurements," Phys. Rev. E 71, 031403 (2005).

${ }^{6}$ R. Soller and S. Koehler, "Drag and lift on rotating vanes in granular beds," Phys. Rev. E 74, 021305 (2006).

${ }^{7}$ H. Katsuragi and D. J. Durian, "Unified force law for granular impact cratering," Nat. Phys. 3, 420-423 (2007).

${ }^{8}$ R. D. Maladen, Y. Ding, C. Li, and D. I. Goldman, "Undulatory swimming in sand: subsurface locomotion of the sandfish lizard," Science 325, 314-318 (2009).

${ }^{9}$ GDR MiDi, “On dense granular flows,” Eur. Phys. J. E 14, 341-365 (2004).

${ }^{10}$ B. Andreotti, Y. Forterre, and O. Pouliquen, Granular Media: Between Fluid and Solid (Cambridge University Press, Cambridge, England, 2013).

${ }^{11}$ R. Candelier and O. Dauchot, "Creep motion of an intruder within a granular glass close to jamming," Phys. Rev. Lett. 103, 128001 (2009).

${ }^{12}$ N. Gravish, P. Umbanhowar, and D. Goldman, "Force and flow transition in plowed granular media," Phys. Rev. Lett. 105, 128301 (2010).

${ }^{13}$ A. Seguin, Y. Bertho, P. Gondret, and J. Crassous, "Dense granular flow around a penetrating object: Experiment and hydrodynamic model," Phys. Rev. Lett. 107, 048001 (2011).

${ }^{14}$ A. Clark, L. Kondic, and R. Behringer, "Particle scale dynamics in granular impact," Phys. Rev. Lett. 109, 238302 (2012).

${ }^{15}$ H. Katsuragi and D. J. Durian, "Drag force scaling for penetration into granular media," Phys. Rev. E 87, 052208 (2013).

${ }^{16}$ K. Wieghardt, "Experiments in granular flow," Annu. Rev. Fluid Mech. 7, 89-114 (1975).

${ }^{17}$ R. Albert, M. Pfeifer, A.-L. Barabási, and P. Schiffer, "Slow drag in a granular medium," Phys. Rev. Lett. 82, 205-208 (1999).

${ }^{18}$ G. Hill, S. Yeung, and S. Koehler, "Scaling vertical drag forces in granular media," Europhys. Lett. 72, 137-143 (2005).

${ }^{19}$ T. A. Brzinski III and D. J. Durian, "Characterization of the drag force in an air-moderated granular bed," Soft Matter 6 , 3038-3042 (2010).

${ }^{20}$ I. Albert, J. Sample, A. Morss, S. Rajagopalan, A.-L. Barabási, and P. Schiffer, "Granular drag on a discrete object: Shape effects on jamming," Phys. Rev. E 64, 061303 (2001).

${ }^{21}$ M. Stone, R. Barry, D. Bernstein, M. Pelc, Y. Tsui, and P. Schiffer, "Local jamming via penetration of a granular medium," Phys. Rev. E 70, 041301 (2004).

${ }^{22}$ D. Costantino, J. Bartell, K. Scheidler, and P. Schiffer, "Low-velocity granular drag in reduced gravity," Phys. Rev. E 83, 011305 (2011).

${ }^{23}$ B. Percier, S. Manneville, J. McElwaine, S. Morris, and N. Taberlet, "Lift and drag forces on an inclined plow moving over a granular surface," Phys. Rev. E 84, 051302 (2011).

${ }^{24}$ Y. Ding, N. Gravish, and D. Goldman, "Drag induced lift in granular media," Phys. Rev. Lett. 106, 028001 (2011). 
${ }^{25}$ F. Q. Potiguar and Y. Ding, "Lift and drag in intruders moving through hydrostatic granular media at high speeds," Phys. Rev. E 88, 012204 (2013).

${ }^{26}$ R. D. Maladen, Y. Ding, P. B. Umbanhowar, A. Kamor, and D. I. Goldman, "Mechanical models of sandfish locomotion reveal principles of high performance subsurface sand-swimming," J. R. Soc., Interface 8(62), 1332-1345 (2011).

${ }^{27}$ C. Li, T. Zhang, and D. I. Goldman, "A terradynamics of legged locomotion on granular media," Science 339, 1408-1412 (2013).

${ }^{28}$ F. Guillard, Y. Forterre, and O. Pouliquen, "Depth-independent drag force induced by stirring in granular media," Phys. Rev. Lett. 110, 138303 (2013).

${ }^{29}$ E. E. de Beer, "Experimental determination of the shape factors and the bearing capacity factors of sand," Géotechnique 20, 387-411 (1970)

${ }^{30}$ C. Kloss, C. Goniva, A. Hager, S. Amberger, and S. Pirker, "Models, algorithms and validation for opensource DEM and CFD-DEM,” Prog. Comput. Fluid. Dyn. 12, 140-152 (2012).

${ }^{31}$ P. Jop, Y. Forterre, and O. Pouliquen, “A constitutive law for dense granular flows," Nature 441, 727-730 (2006).

${ }^{32}$ J. Chauchat and M. Médale, "A three-dimensional numerical model for incompressible two-phase flow of a granular bed submitted to a laminar shearing flow," Comput. Methods Appl. Mech. Eng. 199, 439-449 (2010).

${ }^{33}$ P.-Y. Lagrée, L. Staron, and S. Popinet, "The granular column collapse as a continuum: validity of a two-dimensional Navier-Stokes model with a $\mu(\mathrm{I})$-rheology,” J. Fluid Mech. 686, 378-408 (2011).

${ }^{34}$ K. Kamrin and G. Koval, "Nonlocal constitutive relation for steady granular flow," Phys. Rev. Lett. 108, 178301 (2012).

${ }^{35}$ Y. Fan and K. Hill, "Phase transitions in shear-induced segregation of granular materials," Phys. Rev. Lett. 106, 218301 (2011).

${ }^{36}$ A. Tripathi and D. Khakhar, "Numerical simulation of the sedimentation of a sphere in a sheared granular fluid: A granular stokes experiment," Phys. Rev. Lett. 107, 108001 (2011).

${ }^{37}$ P. A. Cundall and O. D. L. Strack, “A discrete numerical model for granular assemblies," Geotechnique 29, 47-65 (1979).

${ }^{38}$ A. Di Renzo and F. P. Di Maio, "Comparison of contact-force models for the simulation of collisions in DEM-based granular flow codes," Chem. Eng. Sci. 59, 525-541 (2004).

${ }^{39}$ L. Silbert, D. Ertaş, G. Grest, T. Halsey, D. Levine, and S. Plimpton, "Granular flow down an inclined plane: Bagnold scaling and rheology," Phys. Rev. E 64, 051302 (2001).

${ }^{40}$ B. J. Glasser and I. Goldhirsch, "Scale dependence, correlations, and fluctuations of stresses in rapid granular flows," Phys. Fluids 13, 407-420 (2001) 